\title{
A Convergence Rate Result for a Steepest Descent Method and a Minimal Error Method for the Solution of Nonlinear Ill-Posed Problems
}

\author{
A. Neubauer and 0 . Scherzer
}

\begin{abstract}
Recently, convergence and stability of the steepest descent method for the solution of nonlinear ill-posed operator equations have been proven. The same results also hold for the minimal error method. Since for ill-posed problems the convergence of iterative methods may be arbitrarily slow, it is of practical interest to guarantee convergence rates of the iterates under reasonable assumptions. The main emphasis of this paper is to present a convergence rate result in a uniform manner for the steepest descent and the minimal error method for the noise free case.
\end{abstract}

Keywords: Nonlinear ill-posed problems, steepest descent method, minimal error method, regularization methods, discrepancy principle, stopping rule

AMS subject classification: $65 \mathrm{~J} 15,65 \mathrm{~J} 20,47 \mathrm{H} 17$

\section{Introduction}

In this paper the solution of nonlinear operator equations

$$
F(x)=y
$$

by a steepest descent method and a minimal error method is considered. Here $F$ : $\mathcal{D}(F) \rightarrow Y$ with domain $\mathcal{D}(F) \subset X, X$ and $Y$ denote Hilbert spaces with inner products $\langle\cdot, \cdot\rangle$ and norms $\|\cdot\|$, respectively, which can always be identified from the context in which they appear. Throughout this paper we assume attainability of the data $y$, i.e. it is assumed that equation (1.1) has a solution $x$. (which need not be unique). We are mainly interested in problems of the form (1.1) for which the solution $x$ does not depend continuously on the right-hand side data $y$. Such ill-posed problems need to be regularized to obtain reasonable approximations to $x$.

A. Neubauer: Johannes-Kepler-Universität, Institut für Mathematik, Altenberger Str. 69, A 4040 Linz, Austria

O. Scherzer: Johannes-Kepler-Universität, Institut für Mathematik, Altenberger Str. 69, A 4040 Linz, Austria

Partially supported by the Christian Doppler Society (Austria)

ISSN 0232-2064 / \$ 2.50 (C) Heldermann Verlag 
For linear problems the steepest descent method is known to be a regularization method (cf. $[5,6]$ ).

There are several ways of generalizing the steepest descent iteration to nonlinear operator equations (1.1). The one considered in [7] requires a Lipschitz continuous Fréchet derivative $F^{\prime}(\cdot)$ of $F$ in a neighbourhood of $x_{0}$, which we assume in the sequel, and it is defined as follows:

$$
\begin{aligned}
x_{k+1} & =x_{k}+\alpha_{k} s_{k} \quad(k=0,1,2, \ldots) \\
s_{k} & =-F^{\prime}\left(x_{k}\right)^{*}\left(F\left(x_{k}\right)-y\right) \\
\alpha_{k} & =\frac{\left\|s_{k}\right\|^{2}}{\left\|F^{\prime}\left(x_{k}\right) s_{k}\right\|^{2}}
\end{aligned}
$$

where $x_{0}$ is an initial guess which may incorporate a priori knowledge of an exact solution $x_{*}$. It was shown in [7] that this method is a generalization of the well-known steepest descent iteration for the solution of linear ill-posed operator equations: for linear operators $F$, the Fréchet derivative is given by $F^{\prime}\left(x_{k}\right)=F$. Thereforc, the coefficient $\alpha_{k}$ minimizes the norm of the residuum $\left\|F\left(x_{k}+\alpha_{k} s_{k}\right)-y\right\|$ along the searchdirection $s_{k}$. If, alternatively, one chooses $\alpha_{k}$ to minimize $\left\|x_{k}+\alpha_{k} s_{k}-x_{*}\right\|$, one obtains $\alpha_{k}=\left\|F\left(x_{k}\right)-y\right\|^{2} /\left\|s_{k}\right\|^{2}$. The obvious generalization of this minimal error method to the solution of nonlinear problems then yields

$$
\begin{aligned}
x_{k+1} & =x_{k}+\alpha_{k} s_{k} \quad(k=0,1,2, \ldots) \\
s_{k} & =-F^{\prime}\left(x_{k}\right)^{*}\left(F\left(x_{k}\right)-y\right) \\
\alpha_{k} & =\frac{\left\|F\left(x_{k}\right)-y\right\|^{2}}{\left\|s_{k}\right\|^{2}} .
\end{aligned}
$$

If the iteration processes (1.2) and (1.3) are applied to the perturbed problem with $y^{\delta}$ instead of $y$, where

$$
\left\|y-y^{\delta}\right\| \leq \delta
$$

then we write $x_{k}^{\delta}$ for the iterates instead of $x_{k}$. If $y^{\delta}$ does not belong to the range of the operator $F$, then the iterates $x_{k}^{\delta}$ cannot converge but still allow a stable approximation of $x_{*}$ provided the iteration is stopped after an appropriate.number of steps. It was shown in [7] that the steepest descent method (1.2) is convergent, if $F$ satisfies the local property

$$
\left\|F(x)-F(\tilde{x})-F^{\prime}(x)(x-\tilde{x})\right\| \leq \eta\|F(x)-F(\tilde{x})\| \quad\left(\eta<\frac{1}{2}\right)
$$

for all $x, \tilde{x} \in \mathcal{B}_{\rho}\left(x_{0}\right) \subset \mathcal{D}(F)$ and if the iteration is stopped after $k_{*}=k_{*}(\delta)$ iterations according to the generalized discrepancy principle

$$
\left\|F\left(x_{k_{*}}^{\delta}\right)-y^{\delta}\right\| \leq \tau \delta<\left\|F\left(x_{k}^{\delta}\right)-y^{\delta}\right\| \quad\left(0 \leq k<k_{*} ; 2<2 \frac{1+\eta}{1-2 \eta}<\tau\right) .
$$

A detailed interpretation of the assumption above and several examples fulfilling it can be found in $[1,4,8]$. These papers deal with Landweber iteration as a method to 
regularize nonlinear ill-posed problems (1.1). A careful inspection of the proofs in [7] shows that all results proven there are also valid for the minimal error method (1.3).

In [4] convergence rates have been proven for Landweber iteration in the noise free and the perturbed data case, if the operator $F$ satisfies

$$
F^{\prime}(x)=R_{x} F^{\prime}\left(x^{\dagger}\right) \quad\left(x \in \mathcal{B}_{\rho}\left(x_{0}\right)\right)
$$

where $\left\{R_{x}: x \in \mathcal{B}_{\rho}\left(x_{0}\right)\right\}$ is a family of bounded linear operators $R_{x}: Y \rightarrow Y$ with

$$
\left\|R_{x}-I\right\| \leq C\left\|x-x^{\dagger}\right\| \quad\left(x \in \mathcal{B}_{\rho}\left(x_{0}\right)\right)
$$

and $C$ is a positive constant, and if the source condition

$$
x^{\dagger}-x_{0}=\left(F^{\prime}\left(x^{\dagger}\right)^{*} F^{\prime}\left(x^{\dagger}\right)\right)^{\nu} \bar{v} \quad\left(0<\nu \leq \frac{1}{2}\right)
$$

is satisfied; here $x^{\dagger}$ denotes the solution of minimal distance to $x_{0}$, which exists due to (1.4) and (1.5) (compare [4: Proposition 2.1]). Again interpretations of these conditions and examples for which they are true can be found in $[1,4]$. We will show in the next section that the rate

$$
\left\|x_{k}-x^{\dagger}\right\|=O\left(k^{-1 / 2}\right)
$$

is obtained for the steepest descent method and the minimal error method in the noise free case, provided that $F$ is Lipschitz continuously differentiable and that the conditions (1.4) and (1.5) and the source condition

$$
x^{\dagger}-x_{0}=F^{\prime}\left(x^{\dagger}\right)^{*} v
$$

are satisfied; this corresponds to $\nu=\frac{1}{2}$. However, we have not succeeded in proving convergence rates for the case $0<\nu<\frac{1}{2}$; note that there are corresponding results for linear ill-posed problems. In the case of given perturbed data, not even in the special situation of linear operators a convergence rate result is known. Finally, in Section 3 we illustrate conditions (1.4) - (1.6) for a parameter estimation problem.

\section{A convergence rate result}

In [3] $\alpha$-processes for the solution of linear operator equations have been considered. For fixed $\alpha \geq-1$ an $\alpha$-process is defined by

$$
\begin{aligned}
x_{k+1}^{\alpha} & =x_{k}^{\alpha}+\alpha_{k} s_{k} \quad(k=0,1,2, \ldots) \\
s_{k} & =-F^{*}\left(F\left(x_{k}\right)-y\right) \\
\alpha_{k} & =\frac{\left\langle\left(F^{*} F\right)^{\alpha} s_{k}, s_{k}\right\rangle}{\left\langle\left(F^{*} F\right)^{\alpha} s_{k}, F^{*} F s_{k}\right\rangle} .
\end{aligned}
$$

For $\alpha=0$ and $\alpha=-1$ these methods correspond to the steepest descent method (1.2) and the minimal error method (1.3), respectively. The essential idea in proving 
convergence rates of $\alpha$-processes in [3] was to verify that $\left(F^{*} F\right)^{-\nu}\left(x_{k}^{\alpha}-x^{\dagger}\right)$ is bounded if $x^{\dagger}$ satisfies the source condition $x^{\dagger}-x_{0}=\left(F^{*} F\right)^{\nu} v$.

For the steepest descent method and the minimal error method this idea can be carried over to nonlinear problems: we will prove that

$$
\left(F^{\prime}\left(x^{\dagger}\right)^{*} F^{\prime}\left(x^{\dagger}\right)\right)^{-1 / 2}\left(x_{k}-x^{\dagger}\right)
$$

is bounded for both methods, if the source condition (1.6) and the conditions (1.4) and (1.5) are satisfied. Then we proceed with analogous arguments as used in [3] to prove the rate

$$
\left\|x_{k}-x^{\dagger}\right\|=O\left(k^{-1 / 2}\right)
$$

In the next proposition we show that $\left\|x_{k}-x^{\dagger}\right\|$ is monotonically decreasing and that

$$
\alpha_{k}\left\|F\left(x_{k}\right)-y\right\|^{2}=O\left(\left\|x_{k}-x^{\dagger}\right\|^{2}\right)
$$

if $C \rho$ is sufficiently small.

Proposition 2.1. Let conditions (1.4) and (1.5) hold and assume that $C \rho<\frac{4}{7}$. Moreover, assume that $x_{k}$ is obtained either by the steepest descent method (1.2) or by the minimal error method (1.3) and that $x^{\dagger} \in \mathcal{B}_{\rho / 2}\left(x_{0}\right)$. Then $x_{k} \in \mathcal{B}_{\rho / 2}\left(x^{\dagger}\right)$ and

$$
\left\|x_{k+1}-x^{\dagger}\right\|^{2}+\frac{4-7 C \rho}{4-C \rho} \alpha_{k}\left\|F\left(x_{k}\right)-y\right\|^{2} \leq\left\|x_{k}-x^{\dagger}\right\|^{2}
$$

for all $k \in \mathbb{N}_{0}$. Moreover,

$$
\sum_{k=0}^{\infty} \alpha_{k}\left\|F\left(x_{k}\right)-y\right\|^{2}<\infty .
$$

Proof. Let us assume that $x_{k} \in \mathcal{B}_{\rho / 2}\left(x^{\dagger}\right)$. Since $x^{\dagger} \in \mathcal{B}_{\rho / 2}\left(x_{0}\right)$, we then have the inclusion $x_{k} \in \mathcal{B}_{\rho}\left(x_{0}\right)$ and thus relations (1.4) and (1.5) are applicable. Hence we obtain

$$
\begin{aligned}
\| F\left(x_{k}\right)-F\left(x^{\dagger}\right)-F^{\prime} & \left(x_{k}\right)\left(x_{k}-x^{\dagger}\right) \| \\
& =\left\|\int_{0}^{1}\left(F^{\prime}\left(z_{t}\right)-F^{\prime}\left(x_{k}\right)\right)\left(x_{k}-x^{\dagger}\right) d t\right\| \\
& =\int_{0}^{1}\left\|\left(R_{z_{t}}-I+I-R_{x_{k}}\right) F^{\prime}\left(x^{\dagger}\right)\left(x_{k}-x^{\dagger}\right)\right\| d t \\
& \leq \frac{3}{2} C\left\|F^{\prime}\left(x^{\dagger}\right)\left(x_{k}-x^{\dagger}\right)\right\|\left\|x_{k}-x^{\dagger}\right\|
\end{aligned}
$$

where $z_{t}=t x_{k}+(1-t) x^{\dagger} \quad(0 \leq t \leq 1)$. Analogously one verifies that

$$
\left\|F\left(x_{k}\right)-F\left(x^{\dagger}\right)-F^{\prime}\left(x^{\dagger}\right)\left(x_{k}-x^{\dagger}\right)\right\| \leq \frac{1}{2} C\left\|F^{\prime}\left(x^{\dagger}\right)\left(x_{k}-x^{\dagger}\right)\right\|\left\|x_{k}-x^{\dagger}\right\|
$$


holds. This implies

$$
\left(1-\frac{1}{2} C\left\|x_{k}-x^{\dagger}\right\|\right)\left\|F^{\prime}\left(x^{\dagger}\right)\left(x_{k}-x^{\dagger}\right)\right\| \leq\left\|F\left(x_{k}\right)-F\left(x^{\dagger}\right)\right\|=\left\|F\left(x_{k}\right)-y\right\| .
$$

Together with (2.1) this yields

$$
\left\|F\left(x_{k}\right)-F\left(x^{\dagger}\right)-F^{\prime}\left(x_{k}\right)\left(x_{k}-x^{\dagger}\right)\right\| \leq \frac{3 C \rho}{4-C \rho}\left\|F\left(x_{k}\right)-y\right\| .
$$

Thus, we obtain

$$
\begin{aligned}
\| x_{k+1} & -x^{\dagger}\left\|^{2}-\right\| x_{k}-x^{\dagger} \|^{2} \\
= & 2\left\langle x_{k}-x^{\dagger}, x_{k+1}-x_{k}\right\rangle+\left\|x_{k+1}-x_{k}\right\|^{2} \\
= & -2 \alpha_{k}\left\langle x_{k}-x^{\dagger}, F^{\prime}\left(x_{k}\right)^{*}\left(F\left(x_{k}\right)-y\right)\right\rangle+\alpha_{k}^{2}\left\|F^{\prime}\left(x_{k}\right)^{*}\left(F\left(x_{k}\right)-y\right)\right\|^{2} \\
= & \alpha_{k}\left(2\left\langle F\left(x_{k}\right)-y-F^{\prime}\left(x_{k}\right)\left(x_{k}-x^{\dagger}\right), F\left(x_{k}\right)-y\right\rangle-\left\|F\left(x_{k}\right)-y\right\|^{2}\right) \\
& +\alpha_{k}\left(\alpha_{k}\left\|F^{\prime}\left(x_{k}\right)^{*}\left(F\left(x_{k}\right)-y\right)\right\|^{2}-\left\|F\left(x_{k}\right)-y\right\|^{2}\right) \\
\leq & \frac{7 C \rho-4}{4-C \rho} \alpha_{k}\left\|F\left(x_{k}\right)-y\right\|^{2}+\alpha_{k}\left(\alpha_{k}\left\|F^{\prime}\left(x_{k}\right)^{*}\left(F\left(x_{k}\right)-y\right)\right\|^{2}-\left\|F\left(x_{k}\right)-y\right\|^{2}\right) .
\end{aligned}
$$

It remains to be shown that

$$
\alpha_{k}\left\|F^{\prime}\left(x_{k}\right)^{*}\left(F\left(x_{k}\right)-y\right)\right\|^{2} \leq\left\|F\left(x_{k}\right)-y\right\|^{2}
$$

to obtain the asserted estimate. Since $C \rho<\frac{4}{7}$, this estimate implies that $\left\|x_{k}-x^{\dagger}\right\|$ is monotonically decreasing. Together with the inclusion assumption $x^{\dagger} \in \mathcal{B}_{\rho / 2}\left(x_{0}\right)$, an induction argument shows that $x_{k}$ remains in $\mathcal{B}_{\rho / 2}\left(x^{\dagger}\right)$ and that

$$
\sum_{k=0}^{\infty} \alpha_{k}\left\|F\left(x_{k}\right)-y\right\|^{2}<\infty
$$

We will now show that (2.4) holds if $x_{k}$ is obtained by (1.2) and (1.3), respectively. In case of the steepest descent method (1.2) we obtain

$$
\alpha_{k}\left\|F^{\prime}\left(x_{k}\right)^{*}\left(F\left(x_{k}\right)-y\right)\right\|^{2}=\frac{\left\langle F^{\prime}\left(x_{k}\right) s_{k}, F\left(x_{k}\right)-y\right\rangle^{2}}{\left\|F^{\prime}\left(x_{k}\right) s_{k}\right\|^{2}} \leq\left\|F\left(x_{k}\right)-y\right\|^{2}
$$

and in case of the minimal error method (1.3)

$$
\alpha_{k}\left\|F^{\prime}\left(x_{k}\right)^{*}\left(F\left(x_{k}\right)-y\right)\right\|^{2}=\left\|F\left(x_{k}\right)-y\right\|^{2} .
$$

Thus the statement is proved

In order to simplify the notation, we will use the abbreviation

$$
\mathcal{A}=F^{\prime}\left(x^{\dagger}\right)^{*} F^{\prime}\left(x^{\dagger}\right)
$$

in the sequel. 
Lemma 2.2. Let conditions (1.4) and (1.5) hold and assume $C \rho \leq \frac{1}{5}$. Moreover, assume that $x_{k}$ is obtained either by the steepest descent method (1.2) or by the minimal error method (1.3) and that $x^{\dagger} \in \mathcal{B}_{\rho / 2}\left(x_{0}\right)$. If the source condition (1.6) is satisfied, then $\mathcal{A}^{-1 / 2}\left(x_{k}-x^{\dagger}\right)$ is bounded.

Proof. Due to conditions (1.4) and (1.6), an induction argument shows that $x_{k}-$ $x^{\dagger} \in \mathcal{R}\left(\mathcal{A}^{1 / 2}\right)$ for all $k \in N_{0}$. Therefore, we can apply the operator $\mathcal{A}^{-1 / 2}$ both to (1.2) and (1.3) to obtain

$$
\begin{aligned}
&\left\|\mathcal{A}^{-1 / 2}\left(x_{k+1}-x^{\dagger}\right)\right\|^{2}-\left\|\mathcal{A}^{-1 / 2}\left(x_{k}-x^{\dagger}\right)\right\|^{2} \\
&= 2\left\langle\mathcal{A}^{-1 / 2}\left(x_{k}-x^{\dagger}\right), \mathcal{A}^{-1 / 2}\left(x_{k+1}-x_{k}\right)\right\rangle+\left\|\mathcal{A}^{-1 / 2}\left(x_{k+1}-x_{k}\right)\right\|^{2} \\
&=-2 \alpha_{k}\left\langle\mathcal{A}^{-1 / 2}\left(x_{k}-x^{\dagger}\right), \mathcal{A}^{-1 / 2} F^{\prime}\left(x_{k}\right)^{*} \cdot\left(F\left(x_{k}\right)-y\right)\right\rangle \\
&+\alpha_{k}^{2}\left\|\mathcal{A}^{-1 / 2} F^{\prime}\left(x_{k}\right)^{*}\left(F\left(x_{k}\right)-y\right)\right\|^{2} \\
&=-2 \alpha_{k}\left\langle\mathcal{A}^{-1 / 2}\left(x_{k}-x^{\dagger}\right), \mathcal{A}^{-1 / 2} F^{\prime}\left(x^{\dagger}\right)^{*}\left(F\left(x_{k}\right)-y\right)\right\rangle \\
&-2 \alpha_{k}\left\langle\mathcal{A}^{-1 / 2}\left(x_{k}-x^{\dagger}\right), \mathcal{A}^{-1 / 2}\left(F^{\prime}\left(x_{k}\right)^{*}-F^{\prime}\left(x^{\dagger}\right)^{*}\right)\left(F\left(x_{k}\right)-y\right)\right\rangle \\
&+\alpha_{k}^{2}\left\|\mathcal{A}^{-1 / 2} F^{\prime}\left(x_{k}\right)^{*}\left(F\left(x_{k}\right)-y\right)\right\|^{2} \\
&=-2 \alpha_{k}\left\langle F^{\prime}\left(x^{\dagger}\right) \mathcal{A}^{-1}\left(x_{k}-x^{\dagger}\right), F\left(x_{k}\right)-F\left(x^{\dagger}\right)-F^{\prime}\left(x^{\dagger}\right)\left(x_{k}-x^{\dagger}\right)\right\rangle \\
&-2 \alpha_{k}\left\langle\mathcal{A}^{-1 / 2}\left(x_{k}-x^{\dagger}\right), \mathcal{A}^{-1 / 2} F^{\prime}\left(x^{\dagger}\right)^{*}\left(R_{x_{k}}^{*}-I\right)\left(F\left(x_{k}\right)-y\right)\right\rangle \\
&+\alpha_{k}^{2}\left\|\mathcal{A}^{-1 / 2} F^{\prime}\left(x_{k}\right)^{*}\left(F\left(x_{k}\right)-y\right)\right\|^{2}-2 \alpha_{k}\left\|x_{k}-x^{\dagger}\right\|^{2} .
\end{aligned}
$$

The following estimates will be needed to estimate the right-hand side of (2.5). It follows from estimates (2.2) and (2.3), the inclusion $x_{k} \in \mathcal{B}_{\rho / 2}\left(x^{\dagger}\right)$ and the inequality $C \rho \leq \frac{1}{5}$ that

$$
\begin{aligned}
& \left|\left\langle F^{\prime}\left(x^{\dagger}\right) \mathcal{A}^{-1}\left(x_{k}-x^{\dagger}\right), F\left(x_{k}\right)-F\left(x^{\dagger}\right)-F^{\prime}\left(x^{\dagger}\right)\left(x_{k}-x^{\dagger}\right)\right\rangle\right| \\
& \quad \leq\left\|F^{\prime}\left(x^{\dagger}\right) \mathcal{A}^{-1}\left(x_{k}-x^{\dagger}\right)\right\|\left\|F\left(x_{k}\right)-F\left(x^{\dagger}\right)-F^{\prime}\left(x^{\dagger}\right)\left(x_{k}-x^{\dagger}\right)\right\| \\
& \quad \leq \frac{10}{19} C\left\|\mathcal{A}^{-1 / 2}\left(x_{k}-x^{\dagger}\right)\right\|\left\|x_{k}-x^{\dagger}\right\|\left\|F\left(x_{k}\right)-y\right\| .
\end{aligned}
$$

Condition (1.5) implies

$$
\begin{aligned}
& \left|\left\langle\mathcal{A}^{-1 / 2}\left(x_{k}-x^{\dagger}\right), \mathcal{A}^{-1 / 2} F^{\prime}\left(x^{\dagger}\right)^{*}\left(R_{x_{k}}^{*}-I\right)\left(F\left(x_{k}\right)-y\right)\right\rangle\right| \\
& \quad \leq C\left\|\mathcal{A}^{-1 / 2}\left(x_{k}-x^{\dagger}\right)\right\|\left\|\mathcal{A}^{-1 / 2} F^{\prime}\left(x^{\dagger}\right)^{*}\right\|\left\|x_{k}-x^{\dagger}\right\|\left\|F\left(x_{k}\right)-y\right\| \\
& \quad=C\left\|\mathcal{A}^{-1 / 2}\left(x_{k}-x^{\dagger}\right)\right\|\left\|x_{k}-x^{\dagger}\right\|\left\|F\left(x_{k}\right)-y\right\| .
\end{aligned}
$$

Proposition 2.1, conditions (1.4) and (1.5), and inequality $C \rho \leq \frac{1}{5}$ imply

$$
\begin{aligned}
\alpha_{k}^{2}\left\|\mathcal{A}^{-1 / 2} F^{\prime}\left(x_{k}\right)^{*}\left(F\left(x_{k}\right)-y\right)\right\|^{2} & =\alpha_{k}^{2}\left\|\mathcal{A}^{-1 / 2} F^{\prime}\left(x^{\dagger}\right)^{*} R_{x_{k}}^{*}\left(F\left(x_{k}\right)-y\right)\right\|^{2} \\
& \leq\left(1+C\left\|x_{k}-x^{\dagger}\right\|\right)^{2} \alpha_{k}^{2}\left\|F\left(x_{k}\right)-y\right\|^{2} \\
& \leq \frac{23}{13} \alpha_{k}\left\|x_{k}-x^{\dagger}\right\|^{2} .
\end{aligned}
$$


Setting $z_{k}=\left\|\mathcal{A}^{-1 / 2}\left(x_{k}-x^{\dagger}\right)\right\|$, we now obtain with (2.5) - (2.8)

$$
z_{k+1}^{2}-z_{k}^{2} \leq \frac{58}{19} C \alpha_{k}\left\|x_{k}-x^{\dagger}\right\|\left\|F\left(x_{k}\right)-y\right\| z_{k}-\frac{3}{13} \alpha_{k}\left\|x_{k}-x^{\dagger}\right\|^{2}
$$

Using the inequality

$$
2 a b \leq a^{2}+b^{2} \quad(a, b \in \mathbb{R})
$$

with

$$
a=\sqrt{\frac{3}{13} \alpha_{k}}\left\|x_{k}-x^{\dagger}\right\| \quad \text { and } \quad b=\frac{29}{19} C \sqrt{\frac{13}{3} \alpha_{k}}\left\|F\left(x_{k}\right)-y\right\| z_{k}
$$

this implies

$$
z_{k+1}^{2} \leq\left(1+11 C^{2} \alpha_{k}\left\|F\left(x_{k}\right)-y\right\|^{2}\right) z_{k}^{2}
$$

and hence by induction

$$
z_{n}^{2} \leq z_{0}^{2} \prod_{k=0}^{n-1}\left(1+11 C^{2} \alpha_{k}\left\|F\left(x_{k}\right)-y\right\|^{2}\right)
$$

The assertion is proven, if we can show

$$
\prod_{k=0}^{\infty}\left(1+11 C^{2} \alpha_{k}\left\|F\left(x_{k}\right)-y\right\|^{2}\right)<\infty
$$

It is an analysis exercise to show that this is equivalent to

$$
\sum_{k=0}^{\infty} \alpha_{k}\left\|F\left(x_{k}\right)-y\right\|^{2}<\infty
$$

Hence the assertion follows' from Proposition 2.1

Now we can prove the main result of this paper.

Theorem 2.3. Let conditions (1.4) and (1.5) hold and assume $C \rho \leq \frac{1}{5}$. Moreover, assume that $x_{k}$ is obtained either by the steepest descent method (1.2) or by the minimal error method (1.3) and that $x^{\dagger} \in \mathcal{B}_{\rho / 2}\left(x_{0}\right)$. If the source condition (1.6) is satisfied, then we obtain the rate

$$
\left\|x_{k}-x^{\dagger}\right\|=O\left(k^{-1 / 2}\right) \text {. }
$$

Proof. Without loss of generality we can assume $F^{\prime}\left(x^{\dagger}\right) \neq 0$, since otherwise $x^{\dagger}=$ $x_{0}$ and there were nothing to prove. We show that for both methods $\alpha_{k}$ is bounded from below. Conditions (1.2) - (1.5) and inequality $C \rho \leq \frac{1}{5}$ imply

$$
\alpha_{k} \geq\left\|F^{\prime}\left(x_{k}\right)\right\|^{-2} \geq \frac{100}{121}\left\|F^{\prime}\left(x^{\dagger}\right)\right\|^{-2}
$$


If (1.6) holds, this together with Proposition 2.1, estimate (2.3), inequality $C \rho \leq \frac{1}{5}$ and the setting $w_{k}=\mathcal{A}^{-1 / 2}\left(x_{k}-x^{\dagger}\right)$ and $\varepsilon=\frac{247}{484}\left\|F^{\prime}\left(x^{\dagger}\right)\right\|^{-2}$ implies

$$
\begin{aligned}
\left\|\mathcal{A}^{1 / 2} w_{k}\right\|^{2}-\left\|\mathcal{A}^{1 / 2} w_{k+1}\right\|^{2} & \geq \frac{4-7 C \rho}{4-C \rho} \alpha_{k}\left\|F\left(x_{k}\right)-y\right\|^{2} \\
& \geq \varepsilon\left\|F^{\prime}\left(x^{\dagger}\right)\left(x_{k}-x^{\dagger}\right)\right\|^{2} \\
& =\varepsilon\left\langle\mathcal{A}^{3 / 2} w_{k}, \mathcal{A}^{1 / 2} w_{k}\right\rangle
\end{aligned}
$$

An application of Lemma 2 in [3] now yields

$$
\left\|x_{k}-x^{\dagger}\right\| \leq \sqrt{3}\left\|w_{k}\right\|^{2 / 3} \varepsilon^{-1 / 2}\left(\sum_{i=0}^{k-1}\left\|w_{i}\right\|^{-2 / 3}\right)^{-1 / 2}
$$

Since, due to Lemma $2.2,\left\|w_{k}\right\|$ is bounded, this implies the asserted rate

\section{A parameter estimation problem}

In this section we illustrate conditions (1.4) - (1.6) for the following parameter estimation problem. We want to estimate $c$ in

$$
\begin{aligned}
-\Delta u+c u=f & \text { in } \Omega . \\
u=g & \text { in } \partial \Omega
\end{aligned}
$$

where $\Omega$ is either a bounded domain in $\mathbb{R}^{2}$ or $\mathbb{R}^{3}$ with smooth boundary or a parallelepiped, $f \in L^{2}(\Omega)$ and $g \in H^{3 / 2}(\partial \Omega)$.

The nonlinear operator $F: \mathcal{D}(F) \subseteq L^{2}(\Omega) \rightarrow L^{2}(\Omega)$ is defined as the parameterto-solution mapping

$$
F(c)=u(c)
$$

where $u(c)$ is the solution of problem (3.1). One can show (cf., e.g., [2]) the existence of a $\gamma>0$ such that $F$ is well defined on

$$
\mathcal{D}(F)=\left\{c \in L^{2} \mid\|c-\hat{c}\| \leq \gamma \text { for some } \hat{c} \text { with } \hat{c} \geq 0 \text { a.e. }\right\}
$$

It can be argued that the Fréchet derivative and its adjoint are given by

$$
\begin{aligned}
F^{\prime}(c) h & =-A(c)^{-1}(h u(c)) \\
F^{\prime}(c)^{*} v & =-u(c) A(c)^{-1} v
\end{aligned}
$$

with $A(c): H^{2} \cap H_{0}^{1} \rightarrow L^{2}$ defined by

$$
A(c) u=-\Delta u+c u
$$


If $u(c) \geq \kappa(\kappa>0)$ for all $c \in \mathcal{B}_{\rho}\left(c_{0}\right)(\rho \leq \gamma)$, then one can show that

$$
F^{\prime}(d)=R_{d}(c) F^{\prime}(d) \quad\left(c, d \in \mathcal{B}_{\rho}\left(c_{0}\right)\right)
$$

with

$$
R_{d}(c)^{*} w=A(c)\left[\frac{u(d)}{u(c)} A(d)^{-1} w\right]
$$

and

$$
\left\|R_{d}(c)-I\right\| \leq C\|d-c\| \quad\left(c, d \in \mathcal{B}_{\rho}\left(c_{0}\right)\right)
$$

where $C$ is a positive constant independent of $c$ and $d$. Thus conditions (1.4) and (1.5) are satisfied. Moreover, we see from the representation of $F^{\prime}(c)^{*}$ above that the source condition (1.6) is satisfied if and only if $\frac{c^{\dagger}-c_{0}}{u\left(c^{\dagger}\right)} \in H^{2} \cap H_{0}^{1}$.

\section{References}

[1] Binder, A., Hanke, M. and and O. Scherzer: On the Landweber iteration for nonlinear ill-posed problems (submitted).

[2] Colonius, F. and K. Kunisch: Stability for parameter estimation in two-point boundary value problems. J. Reine Angew. Math. 370 (1986), 1 - 29.

[3] Gilyazov, S. F.: Iterative solution methods for inconsistent linear equations with nonselfadjoint operators. Moscow Univ. Comp. Math. and Cyb. 1 (1977), 8 - 13.

[4] Hanke, M., Neubauer, A. and O. Scherzer: A convergence analysis of the Landweber iteration for nonlinear ill-posed problems. Numer. Math. (to appear).

[5] Kammerer, W. J. and M. Z. Nashed: Steepest descent for singular linear operators with nonclosed range. Appl. Anal. 1 (1971), 143 - 159.

[6] McCormick, S. F. and G. H. Rodrigue: $A$ uniform approach to gradient methods for linear operator equations. J. Math. Anal. Appl. 49 (1975), 275 - 285.

[7] Scherzer, O.: A convergence analysis of a method of steepest descent and a two-step algorithm for nonlinear ill-posed problems (submitted).

[8] Scherzer, O.: Convergence criteria of iterative methods based on Landweber iteration for solving nonlinear problems. J. Math. Anal. Appl. (to appear).

Received 07.09.1994; in revised form 21.12.1994 